\title{
Linking Structural Properties with Functionality in Solar Cell Materials - The Effective Mass and Effective Density of States
}

\author{
Thomas Kirchartz ${ }^{1,2}$ and Uwe Rau ${ }^{1}$ \\ ${ }^{1}$ IEK5-Photovoltaics, Forschungszentrum Jülich, 52425 Jülich, Germany \\ ${ }^{2}$ Faculty of Engineering and CENIDE, University of Duisburg-Essen, Carl-Benz-Str. 199, 47057 Duisburg, \\ Germany
}

\begin{abstract}
Understanding crucial material properties making a certain material suitable for a specific application requires a clear methodology linking microscopic parameters with macroscopic functionality. This paper provides such a methodology for the relevant example of the effective mass or effective density of states in semiconductors used as photovoltaic absorber materials. This example is inspired by the recent suggestion that low effective masses are a crucial ingredient explaining the high efficiency and in particular the high open-circuit voltages in metal-halide perovskite solar cells. We critically examine this claim and discuss the conditions that affect the relation between basic properties of a band structure with photovoltaic or more generally optoelectronic functionality. While low effective masses may indeed have a beneficial effect on the open-circuit voltage in the non-radiative limit, using low effective masses as a generic criterion for high optoelectronic functionality would be an oversimplification. Instead the effective mass should always be considered in combination with other material or device specific properties such as the type of band gap (direct or indirect), the doping level and the dominant carrier scattering mechanism.
\end{abstract}




\section{Introduction}

During recent years, metal-halide perovskites have written an unprecedented success story as materials for solar cells ${ }^{1-6}$ and light-emitting diodes.; ${ }^{2 ;-12}$ Achieving solar cell efficiencies above $20 \%{ }^{13-16}$ within less than 5 years of development naturally puts the question on the agenda whether very specific properties ${ }^{17 ; 18}$ give metal-halide perovskites an advantage over other materials that have been studied for decades with only few material technologies achieving the same efficiency level as the perovskites. ${ }^{19}$

Furthermore, important lessons learned from the metal-halide perovskites may help to develop general design principles for a successful systematic search for new photovoltaic absorber materials. Nowadays, computational materials research enables us to systematically scan entire material classes by first principle calculations for promising candidates optimally suited for various applications, e.g. as photovoltaic absorber materials. ${ }^{20-22}$ However, the success of such a computational approach critically relies on the correct definition ${ }^{23}$ of target properties allowing distinction of hopeful candidates from the hopeless ones. ${ }^{24 ; 25}$

Recently, the low effective mass ${ }^{26}$ and consequently the low density of electronic states in the valence band and conduction band found experimentally ${ }^{27 ; 28}$ and theoretically ${ }^{29-31}$ for lead halide perovskites has been named as a possible ingredient to the success of metalhalide perovskites. ${ }^{32}$ In addition, the band structure may involve direct and indirect transitions in a very close energy range which has also been claimed to be beneficial for photovoltaic efficiency. ${ }^{33-36}$ Given that the effective mass or effective density of states are features of the band structure that are always calculated in the context of computational material screening efforts, it is of huge relevance for the community investigating novel materials for photovoltaics, whether these basic features of semiconductor band structures would be beneficial for photovoltaics at least on the level of theory where parabolic approximations to the energy vs. momentum relations of the bands are sensible. A detailed look at the key properties of solar cell absorber material, i.e. absorption, charge transport and recombination 
reveals that the effective mass or effective density of states enters a large number of equations that potentially affect device performance. Thus, the purpose of this manuscript is to start a systematic discussion of the effect of the key features of simple parabolic bands on photovoltaic materials. A closer look at the influence of band structure on absorption and nonradiative recombination quickly reveals that performance depends on whether the semiconductor is direct or indirect as well as whether the material is doped or intrinsic. Thus, in order to achieve a systematic discussion of the effects, we distinguish between these four combinations of a direct/indirect and doped/intrinsic semiconductor for all our simulations. The key results of our study are that in three out of four cases, low effective masses would indeed lead to slightly higher efficiencies at infinite mobilities. However, these higher efficiencies would be reached at substantially higher optimum thicknesses and depending on the exact scattering mechanism dominating mobility, higher effective masses might be often be more beneficial in realistic situations of finite mobilities and ideally low absorber thicknesses.

\section{Definitions of effective mass and effective DOS}

The purpose of this paper is to study the relation between structural properties and device functionality on a level of abstraction that allows us to draw conclusions as a function of a limited number of parameters. The methodology that we establish will then also be transferrable to situations with a reduced level of abstraction. Thus, we will assume that we can treat our semiconducting photovoltaic absorber material of interest by approximating the energy vs. momentum relation of its conduction and valence band with a parabola. In the following, we will briefly repeat the relevant equations that can also be found in various textbooks (see e.g. ref. ${ }^{37}$ ). Near the conduction band edge the relationship between the energy $E$ and momentum $k$ of an electron in the conduction band can be approximated by 


$$
E(k)-E_{\mathrm{C}}=\frac{\mathrm{h}^{2} k^{2}}{2 m_{\mathrm{eff}}} .
$$

where $\mathrm{h}=h / 2 \pi$ is the reduced Planck constant and $m_{\text {eff }}$ is the effective mass (in this case of the electrons in the conduction band). Thus, the effective mass follows from the second derivative of $E$ with respect to the wave vector $k$. Because the curvature of $E(k)$ might be different for different directions in $k$-space the effective mass in general is tensorial. The effective density of states can be calculated via

$$
N_{\mathrm{C}}=2\left(\frac{2 \pi m_{\mathrm{de}} k T}{h^{2}}\right)^{3 / 2} M_{C}
$$

where $k T$ is the thermal energy, $m_{\mathrm{de}}=\left(m_{\mathrm{eff}, 1} m_{\mathrm{eff}, 2} m_{\mathrm{eff}, 3}\right)^{1 / 3}$ is the density of states effective mass and $m_{\text {eff,i }}$ are the effective masses along the principal axes of the ellipsoidal energy surface. The number of equivalent minima in the conduction band is denoted by $M_{\mathrm{C}}$.

Analogous equations can of course be written down for the valence band effective hole mass and effective density of states. For the purpose of this paper, we will restrict ourselves to the simplest case, where the effective mass is the same in all directions of $k$-space, where it is the same for the conduction and valence band. In this simplified situation, we can just talk of effective mass and effective density of states without distinguishing the direction of $k$-space or the band. We also note that while many semiconductors have dispersion relations that become non-parabolic away from the conduction band minimum or valence band maximum, most of the parameters we discuss here are most strongly related to the region of the band very close to the band edges. For instance, the effective density of states is related to the energy dependent density of states close (within a few $k T$ ) to the band edges. The absorption coefficient for a material suitable for high efficiency photovoltaics should increase within a few $k T$ above the band gap to values that lead to complete absorption. Thus, even if the parabolic approximation is unsuitable over a range of hundreds of $\mathrm{meV}$, it would not or not strongly affect the discussion using parabolic bands. Anisotropy in effective masses would 
affect the results but it would still be relatively straightforward to adapt the methodology to more complicated dispersion relations, while it would be outside the scope of the present work to discuss all possible variations of effective mass anisotropy or variations between conduction and valence band effective masses.

\section{Classical arguments in favor of low effective masses}

There are two classical arguments in favour of low effective masses and densities of states. The first argument is based on the relation between the effective density of states and the open-circuit voltage $V_{\text {OC. }}$ For the case of a position independent splitting between the quasi-Fermi energies $E_{\mathrm{fn}}$ and $E_{\mathrm{fp}}$ for electrons and holes it is fair to write $\mathrm{e}^{32}$

$$
\begin{aligned}
& q V_{O C}=E_{F n}-E_{F p}=k T \ln \left[\frac{n p}{n_{0} p_{0}}\right] . \\
& \approx E_{g}+k T \ln \left[\frac{n p}{N_{C} N_{V}}\right]
\end{aligned}
$$

In Eq. (3), $q$ is the elementary charge, $n, p$ are the concentrations and $n_{0}, p_{0}$ the equilibrium concentrations of electrons and holes. The product $n_{0} p_{0}$ is connected to the intrinsic carrier concentration $n_{i}$ and to the effective densities $N_{\mathrm{C}}$ and $N_{\mathrm{V}}$ of states in the conduction and valence band via

$$
n_{0} p_{0}=n_{i}^{2}=N_{C} N_{V} \exp \left[-\frac{E_{g}}{k T}\right]=N_{\text {eff }}^{2} \exp \left[-\frac{E_{g}}{k T}\right] \text {. }
$$

A cursory look at Eqs. (3) and (4) might suggest that a lower effective density of states implies a higher $V_{\mathrm{OC}}$ by tacitly assuming a constant $n p$ product for the comparison. ${ }^{32}$

This is fair if everything else was kept constant including in particular the absorption coefficient. However, also the absorption coefficient is a function of the available density of states for the optical transitions. Because the product $n p$ is a result of the photogeneration and 
recombination of free charge carriers this will affect $V_{\mathrm{OC}}$ and also other photovoltaic properties (like the short-circuit current).

The second frequently made comment about the beneficial influence of the effective mass is based on the idea that larger effective masses reduce the mobility. This is indeed typically the case but depends on the scattering mechanism dominating the mobility in a particular material in the temperature range of interest. However, higher absorption coefficients for higher effective masses would require lower thicknesses for complete absorption and lower thicknesses in turn would require lower mobilities for complete charge carrier collection. Thus, it is not a-priori clear whether low effective mass materials are generally better than high effective mass materials but instead a more detailed and quantitative analysis is required.

\section{Parameters affected by the effective mass or density of states}

In the following, we will briefly discuss how the effective mass affects the key properties of any photovoltaic absorber material, namely the absorption coefficient $\alpha$, the lifetime $\tau$ or recombination rate $R$ and the mobility $\mu$. Table I provides the key relationships that hold on the level of theory, discussed here, where parabolic bands are still appropriate. Table I uses proportionality signs to highlight the effect of effective mass or density of states and to reduce the level of complexity. Table SI in the supporting information provides the same information but now without proportionality signs, also discussing the prefactors as far as this is possible.

The absorption coefficient $\alpha$ depends on the density of available states - linearly for a direct semiconductor ( $\alpha_{\text {dir }} \propto N_{\text {eff }}$ ) and on the product of the densities of states of valence and

conduction band for an indirect semiconductor ( $\alpha_{\text {ind }} \propto N_{\text {eff }}^{2}$ ). The influence of the effective density of states is stronger for indirect than for direct semiconductors because the $k$-selection 
rule is relaxed for indirect transitions due to the influence of phonons. In addition, the absorption coefficient is proportional to the squared momentum matrix element $p_{C V}^{2}$ that is given for simple parabolic bands by $p_{C V}^{2}=m E_{\mathrm{g}} / 2 \times\left(1+m / m_{\text {eff }}\right),{ }^{38}$ where $m$ is the free electron mass. These two effects combined yield the proportionalities given in table I.

Radiative recombination is linked to absorption by the principle of detailed balance. The radiative recombination rate is typically written as $R_{\mathrm{rad}}=B\left(n p-n_{0} p_{0}\right)$ where $B$ denotes the radiative recombination coefficient. The thermal equilibrium value $B n_{0} p_{0}$ of the radiative recombination rate is linked to the absorption coefficient $\alpha$ via the van Roosbroeck-Shockley relation ${ }^{39}$

$$
B n_{0} p_{0}=\int 4 \pi n_{r}^{2} \alpha(E) \phi_{\mathrm{bb}}(E) d E
$$

where $n_{\mathrm{r}}$ is the refractive index of the photovoltaic absorber material and

$$
\phi_{\mathrm{bb}}=\frac{2 E^{2}}{h^{3} c^{2}} \exp \left(-\frac{E}{k T}\right)
$$

denotes the dependence of the black body radiation flux on photon energy $E$. The dependence of the radiative recombination coefficient on the density of states is then given by

$$
B_{\text {dir }} \propto \frac{\left(1+m / m_{\text {eff }}\right)}{N_{\text {eff }}}
$$

for a direct and

$$
B_{\text {ind }} \propto\left(1+\frac{m}{m_{\text {eff }}}\right),
$$

for indirect semiconductors.

In the next step, we consider non-radiative recombination via Shockley-Read-Hall (SRH) recombination ${ }^{40 ; 41}$ with the recombination rate

$$
R_{\mathrm{SRH}}=\frac{n p-n_{0} p_{0}}{\left(n+n_{1}\right) \tau_{p}+\left(p+p_{1}\right) \tau_{n}}
$$


For recombination via deep mid-gap states the reference concentrations $n_{1}$ and $p_{1}{ }^{42}$ are small compared to the free carrier concentrations. For low-level injection conditions we have $n>>n_{1}, n_{0}$ and $p=p_{0}=N_{d}>>p_{1}$, $n$, i.e., the hole concentration (assumed to be the majority carrier concentration without loss of generality) equals the doping concentration $N_{\mathrm{d}}$. The dependence of the $\mathrm{SRH}$ recombination rate on the split of the quasi-Fermi levels $E_{\mathrm{Fn}}-E_{\mathrm{Fp}}$ is then given by

$$
R_{\mathrm{SRH}}^{\text {low }} \approx \frac{n}{\tau_{n}}=\frac{n_{i}^{2}}{N_{d} \tau_{n}} \exp \left(\frac{E_{\mathrm{Fn}}-E_{\mathrm{Fp}}}{k T}\right)=\frac{N_{C} N_{V}}{N_{d} \tau_{n}} \exp \left(\frac{-E_{g}}{k T}\right) \exp \left(\frac{E_{\mathrm{Fn}}-E_{\mathrm{Fp}}}{k T}\right) .
$$

i.e. a quadratic proportionality $R_{\mathrm{SRH}}^{\text {low }} \propto N_{\text {eff }}^{2}$ in low level injection (doped semiconductor).

For high injection conditions we have $n>>n_{1}, n_{0}, p>>p_{1}, p_{0}$, and $n \approx p$ (charge neutrality). With these assumptions we get

$$
\begin{aligned}
& R_{\mathrm{SRH}}^{\text {high }} \approx \frac{n}{\left(\tau_{\mathrm{p}}+\tau_{\mathrm{n}}\right)}=\frac{n_{\mathrm{i}}}{\left(\tau_{\mathrm{p}}+\tau_{\mathrm{n}}\right)} \exp \left(\frac{E_{\mathrm{Fn}}-E_{\mathrm{Fp}}}{2 k T}\right) \\
& =\frac{\sqrt{N_{\mathrm{C}} N_{\mathrm{V}}}}{\left(\tau_{\mathrm{p}}+\tau_{\mathrm{n}}\right)} \exp \left(\frac{-E_{g}}{2 k T}\right) \exp \left(\frac{E_{\mathrm{Fn}}-E_{\mathrm{Fp}}}{2 k T}\right)
\end{aligned}
$$

In contrast to the low level case, SRH under high level injection is an 'ideality two' process (i.e. $R_{\mathrm{SRH}}^{\text {high }} \propto \exp \left(\left[E_{F n}-E_{F p}\right] /[2 k T]\right)$ ) and it is linearly dependent on the effective density of states $\left(R_{\mathrm{SRH}}^{\text {high }} \propto N_{\text {eff }}\right)$ and not quadratically as the recombination under low level injection. Note that the Shockley-Read-Hall lifetimes themselves do not depend on effective mass if we assume a multiphonon transition between band and trap state as discussed in ref. ${ }^{43}$. The assumptions on which this statement is based are discussed in more detail in the supplemental material.

Having summed up the dependence of the basic photovoltaic actions, i.e. light absorption as well as radiative and non-radiative recombination, on the density of states at the edges at 
the conduction and valence band we are now able to calculate the influence of these densities of states on the efficiency potential of the solar cell using a basic detailed balance model. ${ }^{24}$

\section{Efficiency calculations}

a) Radiative efficiencies

To calculate the efficiency potential from the given material parameters we follow the method outlined in Ref. ${ }^{24}$. In a first step, we will calculate the radiative efficiency for high mobilities and in section (b), we will look at the efficiency in the presence of SRH recombination but still for high mobilities. Incomplete charge collection due to low mobilities is then discussed in section VII.

The conversion efficiency $\eta$ is given by ${ }^{44}$

$$
\eta=\frac{J_{\mathrm{SC}} V_{\mathrm{OC}} F F}{P_{\text {sun }}}
$$

where $J_{\mathrm{SC}}$ denotes the short circuit current density, $V_{\mathrm{OC}}$ the open circuit voltage, $F F$ the fill factor, and $P_{\text {sun }}$ the power density of the incident sun light. The open-circuit voltage is given by

$$
V_{\mathrm{OC}}=\frac{n_{\mathrm{id}} k T}{q} \ln \left(\frac{J_{\mathrm{SC}}}{J_{0}}+1\right)
$$

where $n_{\mathrm{id}}$ is the ideality factor of the dominant recombination process. The short circuit current density is given by

$$
J_{\text {SC }}=\int_{E g}^{\infty} A(E) \phi_{\text {sun }}(E) d E
$$

where $A$ is the absorptance of the solar cell and $\phi_{\text {sun }}$ denotes the solar spectrum as tabulated in Ref. ${ }^{45}$. The saturation current density $J_{0}$ depends on the recombination process. For 
radiative recombination we can calculate $J_{0}^{\mathrm{rad}}$ from the absorptance of the cell ${ }^{46 ; 47}$ according to

$$
J_{0}^{\mathrm{rad}}=\int_{E g}^{\infty} A(E) \phi_{\text {cell }}(E) d E
$$

where $\phi_{\text {cell }}=\pi \phi_{\mathrm{bb}}$ is the black body emission at the cell's temperature $T=300 \mathrm{~K}$.

Note that for the calculation of the absorptance spectrum $A(E)$ from a given absorption coefficient $\alpha(E)$ we need to make an assumption of the specific light trapping structure. ${ }^{24}$ In the present work we refer to a Lambertian light trapping scheme for all calculations with the equations given in Ref. ${ }^{48}$ which has proven in the past to be a useful standard to compare the photovoltaic potential of different photovoltaic absorber materials. ${ }^{24}$ We assume for the moment that the carrier mobilities are high enough to warrant perfect carrier collection implying the electron and hole Fermi-levels are flat throughout the absorber for any bias condition. Furthermore, we consider the situation where the optical absorption and emission is strictly limited to energies above the band gap energy $E_{\mathrm{g}}$.

\section{b) Efficiencies in the presence of non-radiative recombination}

While efficiency and $J_{\mathrm{SC}}$ are calculated using the same equations as for the radiative limit, we have to use a different saturation current density. In the following, we will restrict ourselves to the simple case, where the lifetimes for electrons and holes are the same, i.e. $\tau_{n}=\tau_{p}=\tau$. The recombination current in the limit of high mobilities is given by $J=q R d$, where the recombination rate $R=n / \tau$ in low level injection (eq. (10)) and $d$ is the thickness. The saturation current density is given as the voltage independent prefactor and can thus be written in low level injection (doped semiconductor) as

$$
J_{0}^{\text {low }}=q d \frac{n_{0}}{\tau}=q d \frac{N_{\text {eff }}^{2}}{N_{d} \tau} \exp \left(\frac{-E_{g}}{k T}\right)
$$


From Eq. (11) we derive for the saturation current density under high injection conditions

$$
J_{0}^{\mathrm{high}}=q d \frac{\sqrt{N_{C} N_{V}}}{2 \tau} \exp \left(\frac{-E_{g}}{2 k T}\right)
$$

Shockley-Read-Hall recombination at high level injection $(n \approx p)$ is an 'ideality 2' process, implying that the dependence of the recombination current on the external voltage $V$ reads

$$
J_{\mathrm{SRH}}^{\text {high }}=J_{0}^{\text {high }}\left[\exp \left(\frac{q V}{2 k T}\right)-1\right] .
$$

Note that we assume in this paper that bulk recombination is dominating the saturation current density and we neglect surface recombination for simplicity. Note, however, that surface recombination would change the way $J_{0}$ depends on thickness in a rather complicated way. $49 ; 50$

\section{Results for infinite mobilities}

In the following, we use the equations introduced in chapter $\mathbf{V}$ to calculate the efficiencies in the radiative limit and in the presence of non-radiative recombination for the four cases we distinguished above: direct and indirect band gap semiconductors both either for low or high level injection (i.e. high or low doping density). Initially, we assume the mobilities to be sufficiently high so that charge collection is always perfect. The parameter for the optical properties are given in table SII in the supplemental material (columns generic direct and generic indirect) and the SRH lifetime is $\tau_{\mathrm{n}}=\tau_{\mathrm{p}}=1 \mu \mathrm{s}$. Even in this idealized highmobility situation, efficiencies will depend on the thickness of the absorber layers. For low thicknesses, light is not efficiently absorbed and the short-circuit current is reduced. In the radiative limit, this reduction at low thicknesses is the only relevant effect and efficiency therefore just saturates if thicknesses are sufficiently high for the absorptance to resemble the ideal step-function. If non-radiative bulk recombination is dominant, the dark saturation currents increase linearly with thicknesses (c.f. Eqs. (16) and (17)) and therefore, the 
efficiency will start decreasing once the short-circuit current is in saturation. Therefore, in the presence of non-radiative recombination, we expect to observe a finite optimum thickness even for infinite mobilities.

Figure 1 shows the efficiency for the four cases discussed as a function of thickness for five different values of the effective density of states $\left(N_{\text {eff }}=10^{18}, 10^{18.5}, 10^{19}, 10^{19.5}, 10^{20} \mathrm{~cm}^{-}\right.$ $\left.{ }^{3}\right)$. The dashed lines always correspond to the radiative limit. Here, we observe that the saturation value $\eta=30.5 \%$ at high thicknesses is independent of effective DOS and identical to the Shockley-Queisser limit ${ }^{51}$ at a band gap $E_{\mathrm{g}}=1.6 \mathrm{eV}$. This observation is consistent with the Shockley-Queisser limit being independent of internal properties such as effective DOS or effective mass. At lower thicknesses, however, we observe differences that are due to the different absorption coefficients. The higher the effective DOS, the higher the absorption coefficient will be. Therefore lower thickness will be sufficient to absorb the same amount of light and, thus, the efficiency remains high for lower thicknesses as opposed to the cases with lower effective DOS. This is true for all four situations (a)-(d).

In the presence of non-radiative recombination, we observe a decrease of the efficiency relative to the radiative limit which increases towards higher thicknesses. ${ }^{52}$ This increase does not occur in the radiative limit because the increased generation of photons in an increasing absorber volume is compensated by absorption of these photons by the absorber itself (photon recycling). ${ }^{53}$ In the radiative limit, peak efficiencies are always the same (the ShockleyQueisser limit) and efficiencies at lower thicknesses will always be highest whenever the effective DOS is highest (and therefore the absorption coefficient). In contrast, in the presence of non-radiative recombination the trend with effective DOS depends on the mode of comparison. If for instance we focus on Fig. 1a, the case of a direct semiconductor in high level injection (undoped semiconductor), we see that for low thicknesses higher DOS are beneficial. For high thicknesses, however, lower effective DOS are more beneficial because now the effect of DOS on absorption coefficient has become irrelevant while the positive 
effect of effective DOS on recombination is still relevant. In order to summarize the data in Fig. 1 in a way that is independent of the arbitrary choice of a constant thickness, we always determine the optimum efficiency as a function of thickness (marked with symbols in Fig. 1).

Figure 2 presents (a) the efficiencies and (b) the optimum thicknesses calculated for a higher number of effective DOS values. From Fig. 2(a) we see that in three out of four cases, the lower effective DOS values indeed lead to the higher efficiencies. However, these are reached at higher optimum thicknesses as shown in Fig. 2(b). If we compare the four presented cases, we observe that in case of an indirect band gap the higher effective DOS are generally favored as opposed to the direct band gap case. This is due to the stronger

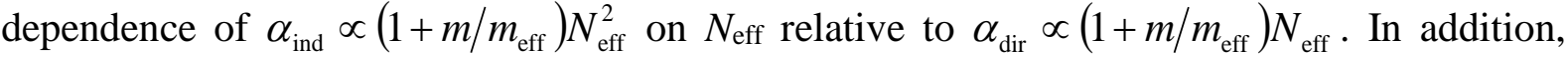
the low injection case (doped) is in most situations (except for indirect band gaps and $N_{\text {eff }}>$ $10^{20} \mathrm{~cm}^{-3}$ ) better than the high injection case (intrinsic). This is due to the low injection case reaching higher fill factors and often also higher open circuit voltages by avoiding the $n_{\mathrm{id}}=2$ recombination process, present in the high injection case. ${ }^{54}$

The product $\alpha d_{\mathrm{opt}}$ of optimum thickness and absorption coefficient is approximately constant as a function of the effective density of states $N_{\text {eff: }}$ An increase in absorption coefficient by a factor of 10 would lead to a factor 10 lower optimum thickness. Note that $\alpha d_{\text {opt }}$ is only independent of $N_{\text {eff }}$ but not of other parameters in the model (like direct. vs. indirect band gap). This leads to, e.g., the short-circuit current density $J_{\mathrm{sc}}$ and the light outcoupling efficiency $p_{\mathrm{e}}$ being roughly constant with $N_{\text {eff }}$ if we always look at the optimum thickness (see Fig. S3 in the supplemental material). If we are sufficiently far away from the radiative limit that we can neglect photon recycling, the external light emitting diode quantum efficiency $Q_{\mathrm{e}}^{\text {lum }}$ is related to the internal quantum efficiency $Q_{\mathrm{i}}^{\text {lum }}$, the ratio between the radiative and the total recombination rate, via ${ }^{53 ; 55}$ 


$$
Q_{\mathrm{e}}^{\text {lum }}=\frac{p_{\mathrm{e}} Q_{\mathrm{i}}^{\text {lum }}}{1-p_{\mathrm{r}} Q_{\mathrm{i}}^{\text {lum }}} \approx p_{\mathrm{e}} Q_{\mathrm{i}}^{\text {lum }} ; Q_{\mathrm{i}}^{\text {lum }}<<1
$$

In addition, the open-circuit voltage $V_{\mathrm{OC}}$ depends on the open-circuit voltage $V_{\mathrm{OC}}^{\mathrm{rad}}$ in the radiative limit via ${ }^{46 ; 55 ; 56}$

$$
\begin{aligned}
& q V_{\mathrm{OC}}=q V_{\mathrm{OC}}^{\mathrm{rad}}+k T \ln \left\{Q_{\mathrm{e}}^{\mathrm{lum}}\right\} \\
& \approx q V_{\mathrm{OC}}^{\mathrm{rad}}+k T \ln \left\{p_{\mathrm{e}} Q_{\mathrm{i}}^{\mathrm{lum}}\right\} .
\end{aligned}
$$

The outcoupling efficiency $p_{\mathrm{e}}$ is a function of thickness as shown in ref. ${ }^{53}$ and can be calculated using eq. (25) in ref. ${ }^{55}$. Thus, we can understand the behavior of $V_{\text {OC }}\left(d_{\text {opt }}\right)$ as shown in Fig. 3a by looking at the trend in $Q_{\mathrm{i}}^{\text {lum }}$ with $d_{\text {opt. }}$ For the case $Q_{\mathrm{i}}^{\text {lum }}<<1$ we have

$$
Q_{\mathrm{i}}^{\text {lum }} \approx \frac{R_{\mathrm{rad}}}{R_{\mathrm{SRH}}}=\frac{B_{\mathrm{rad}} n p}{R_{\mathrm{SRH}}}
$$

where $B_{\text {rad }}$ is the radiative recombination coefficient $\left(B_{\text {rad }}=B_{\text {dir }}\right.$ for a direct recombination coefficient and $B_{\text {rad }}=B_{\text {ind }}$ for an indirect recombination coefficient). Table II summarizes the dependencies of $Q_{i}^{\text {lum }}$ on the densities of states for the four different cases.

Figure $3 \mathrm{~b}$ shows the calculated $Q_{\mathrm{e}}^{\mathrm{lum}}$ (solid lines) and the analytical approximation for $\propto Q_{\mathrm{i}}^{\text {lum }}$ from table II (dashed lines) for the four cases from Figs. 1 and 2. The dashed and solid lines approximately agree with each other but the agreement lacks precision in some cases because $p_{\mathrm{e}}$ may change slightly with $N_{\text {eff }}$ as shown in Fig. S3. This is in particular true for the high injection, indirect band gap case (orange). As expected from Eq. (20), there is an obvious agreement between the trends in $Q_{\mathrm{e}}^{\mathrm{lum}}$ and $Q_{\mathrm{i}}^{\mathrm{lum}}$ (Fig. 3b) and the trends in $V_{\mathrm{OC}}\left(d_{\mathrm{opt}}\right)$ shown in Fig. 3a. Thus, trends in efficiencies (Fig. 2a) and open circuit voltages (Fig.3a) are reasonably well explained by the relations presented in table II, rather than by the naive picture given by Eq. (3). 


\section{Effect of effective mass on charge collection}

One of the key arguments in favor of low effective mass materials is based on the advantageous effect of low effective masses on charge-carrier mobilities. The mobility $\mu$ is proportional to the ratio between the momentum relaxation time $\tau_{\mathrm{m}}$ and the effective mass (i.e. $\left.\mu \propto \tau_{\mathrm{m}} / m_{\text {eff }}\right) .{ }^{57}$ The momentum matrix element depends on the dominant scattering process and there are a range of different dependencies of $\tau_{\mathrm{m}}$ on $m_{\text {eff }}$ as shown in table I. ${ }^{57}$ If we focus on lattice scattering, we observe the cases $\tau_{\mathrm{m}} \propto m_{\mathrm{eff}}^{-1 / 2}$ for polar optical phonons, $\tau_{\mathrm{m}} \propto m_{\mathrm{eff}}^{-3 / 2}$ for acoustical phonons and zero order optical phonons and $\tau_{\mathrm{m}} \propto m_{\mathrm{eff}}^{-5 / 2}$ for first order optical phonons. ${ }^{57}$ However, charge collection not only depends on mobility but also on the lifetime (which in case of non-radiative recombination via phonons is independent of effective mass) and more importantly the thickness of the absorber layer. In case of charge collection within the neutral base of a doped semiconductor, charge collection would depend mostly on the ratio between the diffusion length $L_{\text {diff }}=\sqrt{\mu t k T / q}$ and the thickness $d$ of the absorber layer. $^{44 ; 58 ; 59}$ In contrast, if charge collection was dominated by drift rather than diffusion, it would depend on the ratio between the drift length $L_{\text {drift }}=\mu \tau F$ and the absorber thickness. ${ }^{60-}$ ${ }^{62}$ Here, $F$ is the electric field, which in the absence of strong band bending would be given by $F=\left(V_{\mathrm{bi}}-V\right) / d$, where $V_{\mathrm{bi}}$ is the built-in voltage and $V$ is the applied voltage. Thus, if we only consider the mobility $\mu$ and the optimum thickness $d_{\text {opt }}$ as the two parameters that depend on effective mass, we obtain $L_{\text {diff }} / d_{\text {opt }} \propto \sqrt{\mu / d_{\text {opt }}^{2}}$ and $L_{\text {drift }} / d_{\text {opt }} \propto \mu / d_{\text {opt }}^{2}$. Collection is therefore in first approximation a non-linear function ${ }^{63 ; 64}$ of the ratio $\mu / d_{\mathrm{opt}}^{2}$ independent of what mechanism (drift or diffusion) exactly controls recombination and transport. Thus, we compare in the following how a figure of merit (FOM) for charge collection given by this ratio $\left(F O M=\mu / d_{\mathrm{opt}}^{2}\right)$ depends on effective mass or effective DOS. 
Figure 4 shows the $F O M=\mu / d_{\mathrm{opt}}^{2}$ as a function of the effective DOS for different relations between the mobility and the effective mass: (a) $\mu \propto m_{\text {eff }}^{-3 / 2}$, (b) $\mu \propto m_{\text {eff }}^{-5 / 2}$, and (c) $\mu \propto m_{\text {eff }}^{-7 / 2}$. The value for the $F O M=\mu / d_{\text {opt }}^{2}$ is always normalized to one for $N_{\text {eff }}=10^{18} \mathrm{~cm}^{-3}$. Depending on the ratio between $\mu$ and $m_{\text {eff, }}$ and depending on whether we are dealing with a direct or an indirect band gap, $F O M=\mu / d_{\text {opt }}^{2}$ may be increasing or decreasing with $N_{\text {eff. }}$ The type of band gap has a strong influence on the relation between absorption coefficient and therefore optimum thickness and $N_{\text {eff. }}$ Thus, in case of the indirect band gap semiconductors, higher $N_{\text {eff }}$ are always better, because the lower optimum thickness compensates the decrease in mobility. For the direct band gap cases, the situation is less clear. Here it depends on the relation between $\mu$ and $m_{\text {eff. For }} \mu \propto m_{\text {eff }}^{-3 / 2}$, high $N_{\text {eff }}$ is still more favourable, while for the two other cases ( $\mu \propto m_{\text {eff }}^{-5 / 2}$ and $\mu \propto m_{\text {eff }}^{-7 / 2}$ ) high $N_{\text {eff }}$ leads to lower $F O M=\mu / d_{\text {opt }}^{2}$.

To illustrate the meaning of the $F O M=\mu / d_{\mathrm{opt}}^{2}$ with respect to solar cell efficiencies, we used drift-diffusion simulations using the software ASA ${ }^{65 ; 66}$ for two representative cases. Figure 5a shows the efficiency as a function of thickness for $\mu \propto m_{\mathrm{eff}}^{-3 / 2}$ for two values of the effective DOS ( $N_{\text {eff }}=10^{18}$ and $10^{20} \mathrm{~cm}^{-3}$ ) and the high injection, direct band gap scenario. In addition, we varied the mobility $\mu_{0}$ at $m_{\text {eff }}=m$ to show how sensitive the situations are with respect to lowering the mobility. Figure $5 b$ shows the equivalent simulation for the case $\mu \propto m_{\text {eff }}^{-7 / 2}$. For both Fig. 5a and b, the high mobility cases are approximately identical to Fig. 1a (black and pink line). In case of (a) $\mu \propto m_{\text {eff }}^{-3 / 2}$, Fig. 4 indicates that the $F O M=\mu / d_{\text {opt }}^{2}$ hardly changes with $N_{\text {eff. }}$ Thus, the effect of lowering $\mu_{0}$ is comparable but the whole set of curves is shifted horizontally. In case of (b) $\mu \propto m_{\text {eff }}^{-7 / 2}$, the $F O M=\mu / d_{\text {opt }}^{2}$ decreases strongly with higher $N_{\text {eff }}$ (purple line in Fig. 4c). Thus, in Fig. 5b, the region of high efficiency on the thickness axis is much larger for a given $\mu_{0}$ if the effective DOS is low. The numerical 
simulations also show that the differences in absolute efficiency at the peaks of the curves are relatively small as compared to the differences in efficiency, if the device is slightly too thick or too thin, i.e. out of the optimum region that is defined by the absorption coefficient on the low thickness side and $\mu \tau / d^{2}$ on the high thickness side.

Figure 6 shows the equivalent data to Fig. 5 but for the case of low level injection $\left(N_{\text {dop }}=\right.$ $10^{17} \mathrm{~cm}^{-3}$ ) and an indirect bandgap, i.e. a situation as encountered in crystalline Si solar cells. Now the absorption coefficient is much lower and the optimum thicknesses correspondingly higher. We chose unlike in the rest of the paper a lifetime of $\tau=100 \mu$ s ( $1 \mu$ s everywhere else) to keep the necessary mobilities in a more realistic range. Again, we always show to values of the effective density of states $\left(N_{\text {eff }}=10^{18}\right.$ and $\left.10^{20} \mathrm{~cm}^{-3}\right)$ in each panel, while panel (a) deals with a moderate dependence of mobility on effective mass ( $\mu \propto m_{\text {eff }}^{-3 / 2}$ ), and panel (b) deals with a rather strong dependence of mobility on effective mass $\left(\mu \propto m_{\text {eff }}^{-7 / 2}\right)$. The mobility prefactors used are here $\left(\mu_{0}=10^{-1}, 10^{0}, 10^{1}, 10^{2} \mathrm{~cm}^{2} / \mathrm{Vs}\right)$. Note that the FOMs that correspond to the situation shown in Fig. 6 are given by the green lines in Figs. 4a and 4c. consistent with the trend in the FOMs in Fig. 4, in panel (a), the high effective DOS is substantially more beneficial than the lower DOS, while in panel (b) the high DOS is still better but only by a small margin.

\section{Summary and conclusions}

The first result of the present paper is that there is no simple answer to the question whether high or low densities of states in the conduction or valence band of a photovoltaic absorber material are preferred. The primary photovoltaic interaction, light absorption by the generation of electron hole pairs, is enhanced by a high density of states. In the radiative limit, this advantage is counterbalanced by the fact that radiative recombination is strengthened by high densities of states in the same why as the light absorption. ${ }^{39}$ Thus, materials with a low 
density of states have the same radiative efficiency limit as materials with high densities of states. However, the former attain high efficiencies only at thicknesses that are accordingly larger than those required for the latter materials.

Non-radiative recombination is also promoted by a high density of states. Here we have to consider more details about the type of semiconductor (direct or indirect) and the level of excitation relative to the doping density (high level vs. low level injection). For the most important combinations (direct semiconductors under high injection conditions and indirect semiconductors under low injection conditions) the combined effects, enhanced absorptance vs. enhanced recombination by high density of states, again almost cancel out. However, the proportionality of the internal luminescence efficiency $Q_{i}^{\text {lum }} \propto\left(1+m / m_{\text {eff }}\right)$ for these two cases slightly favors low effective masses (density of states). Thus, the attainable open circuit voltages are slightly higher for materials with a low density of states.

Finally, charge-carrier mobilities decrease for all considered scattering mechanisms with increasing effective densities of states or mass. However, this effect is counterbalanced by the lower required thickness for materials with higher densities of states. In this paper we considered lattice scattering via optical and acoustic phonons which provides a range of proportionalities between mobility and effective mass from $\mu \propto m_{\mathrm{eff}}^{-3 / 2}$ to $\mu \propto m_{\mathrm{eff}}^{-7 / 2}$. Obviously, the cases with $\mu \propto m_{\text {eff }}^{-7 / 2}$ are much more likely to favor low effective masses. In contrast, we also observe substantial differences whether we consider direct or indirect band gaps. For indirect band gaps, the relation between absorption coefficient and therefore also optimum thickness is much stronger than for direct band gaps. Therefore, even for scattering mechanisms leading to $\mu \propto m_{\mathrm{eff}}^{-7 / 2}$ photovoltaic materials with indirect band gaps are more likely to be useful if they have higher effective masses.

From the above findings we conclude that neither high nor low densities of states in the bands of the free carriers are in any way a silver bullet like indicator for the suitability of a 
semiconductor material as a photovoltaic absorber. Thus, other indicators have to be named and these indicators are reflected in the parameters for the non-radiative recombination processes. Clearly, the density of states of the free carriers is not the magic switch, but the density of defects clearly is. Thus, materials that have a high formation enthalpy for deep, recombination active defects are preferred over materials which form a lot of those defects. ${ }^{2 ; 67 ; 68}$ The other possible switch is the capture cross section of such defects. In particular for direct semiconductors the interaction between electrons and phonons via multiphonon recombination events is of huge importance. Material properties, such as phonon energies, ${ }^{69 ; 70}$ the Huang Rhys factor, ${ }^{71 ; 72}$ or the deformation potential should be clearly related to the photovoltaic performance. The second result of the current paper is that it provides the methodological framework and the necessary set of scenarios that allow correlating such microscopic parameters with the photovoltaic potential of specific materials.

\section{Author Information}

Corresponding Author:

To whom correspondence should be addressed. E-Mail: t.kirchartz@fz-juelich.de

There are no conflicts of interest to declare

\section{Acknowledgements}

TK and UR acknowledge support from the DFG (Grant Nos. KI-1571/2-1 and RA 473/7-1). The authors would like to thank Urs Aeberhard (Jülich), David Egger (Regensburg) and Davide Moia (London) for helpful discussions. 
Table I: Relations between the key photovoltaic material parameters for absorption, recombination and transport and the effective mass and effective DOS, respectively. Here only proportionalities are given while the full equations at least in case of absorption and recombination are given and discussed in the supplemental material.

\begin{tabular}{|c|c|}
\hline quantity & $\begin{array}{l}\text { Dependence on effective mass } m_{\text {eff }} \text { or effective DOS } N_{\text {eff }}(m \\
\text { denotes the free electron mass) }\end{array}$ \\
\hline $\begin{array}{l}\text { absorption coefficient } \alpha_{\text {dir }} \\
\text { (direct semiconductor) }\end{array}$ & $\alpha_{\mathrm{dir}} \propto\left(1+\frac{m}{m_{\mathrm{eff}}}\right) N_{\mathrm{eff}} \frac{\left(E-E_{g}\right)^{1 / 2}}{E} 38 ; 73$ \\
\hline $\begin{array}{l}\text { absorption coefficient } \alpha_{\text {ind }} \\
\text { (indirect semiconductor) }\end{array}$ & $\alpha_{\text {ind }} \propto\left(1+\frac{m}{m_{\text {eff }}}\right) N_{\text {eff }}^{2} \frac{\left(E-E_{g}\right)^{2}}{E} 38 ; 74$ \\
\hline $\begin{array}{l}\text { Radiative recombination } \\
\text { coefficient } B_{\text {dir }} \text { (direct } \\
\text { semiconductor) }\end{array}$ & $B_{\mathrm{dir}} \propto \frac{\left(1+m / m_{\mathrm{eff}}\right)}{N_{\mathrm{eff}}}$ \\
\hline $\begin{array}{l}\text { Radiative recombination } \\
\text { coefficient } B_{\text {ind }} \text { (indirect } \\
\text { semiconductor) }\end{array}$ & $B_{\text {ind }} \propto\left(1+\frac{m}{m_{\text {eff }}}\right)$ \\
\hline $\begin{array}{l}\text { SRH recombination rate } R_{\mathrm{SRH}} \\
\text { (low level injection) }\end{array}$ & $R_{\mathrm{SRH}}^{\text {low }} \propto \frac{N_{\text {eff }}^{2}}{N_{\mathrm{d}} \tau_{\min }}$ \\
\hline $\begin{array}{l}\text { SRH recombination rate } R_{\mathrm{SRH}} \\
\text { (high level injection) }\end{array}$ & $R_{\mathrm{SRH}}^{\text {high }} \propto \frac{N_{\mathrm{eff}}}{\tau_{n}+\tau_{p}}$ \\
\hline $\begin{array}{l}\text { Capture cross section (for } \\
\text { multiphonon transitions) }\end{array}$ & $\sigma \propto \sqrt{m_{\mathrm{eff}}} 43 ; 75 ; 76$ \\
\hline Thermal velocity & $v_{\mathrm{th}} \propto 1 / \sqrt{m_{\mathrm{eff}}}$ \\
\hline $\begin{array}{l}\text { SRH lifetime (for } \\
\text { multiphonon transitions) }\end{array}$ & $\tau=\left(\sigma v_{\mathrm{th}} N_{\mathrm{t}}\right)^{-1}=$ const. \\
\hline mobility $\mu$ & $\mu \propto \frac{\tau_{\mathrm{m}}}{m_{\mathrm{eff}}} 57$ \\
\hline $\begin{array}{l}\text { Momentum relaxation time } \\
\text { for lattice scattering }\end{array}$ & $\tau_{\mathrm{m}} \propto\left\{\begin{array}{c}m_{\mathrm{eff}}^{-1 / 2} \text { for polar optical phonons } \\
m_{\mathrm{eff}}^{-3 / 2} \text { for acoustical and zero - order optical phonons }{ }^{57} \\
m_{\mathrm{eff}}^{-5 / 2} \text { for first order optical phonons }\end{array}\right.$ \\
\hline
\end{tabular}


Table II: Internal LED quantum efficiency $Q_{i}^{\text {lum }}$ as a function of effective mass or effective DOS for the four cases discussed in the paper (high level injection vs. low level injection and direct band gap vs. indirect band gap).

\begin{tabular}{lll}
\hline & High level injection & Low level injection \\
\hline Direct gap & $Q_{\mathrm{i}}^{\mathrm{lum}} \propto\left(1+m / m_{\mathrm{eff}}\right)$ & $Q_{\mathrm{i}}^{\mathrm{lum}} \propto \frac{\left(1+m / m_{\mathrm{eff}}\right)}{N_{\text {eff }}}$ \\
Indirect gap & $Q_{\mathrm{i}}^{\mathrm{lum}} \propto\left(1+m / m_{\mathrm{eff}}\right) N_{\text {eff }}$ & $Q_{\mathrm{i}}^{\mathrm{lum}} \propto\left(1+m / m_{\mathrm{eff}}\right)$ \\
\hline
\end{tabular}




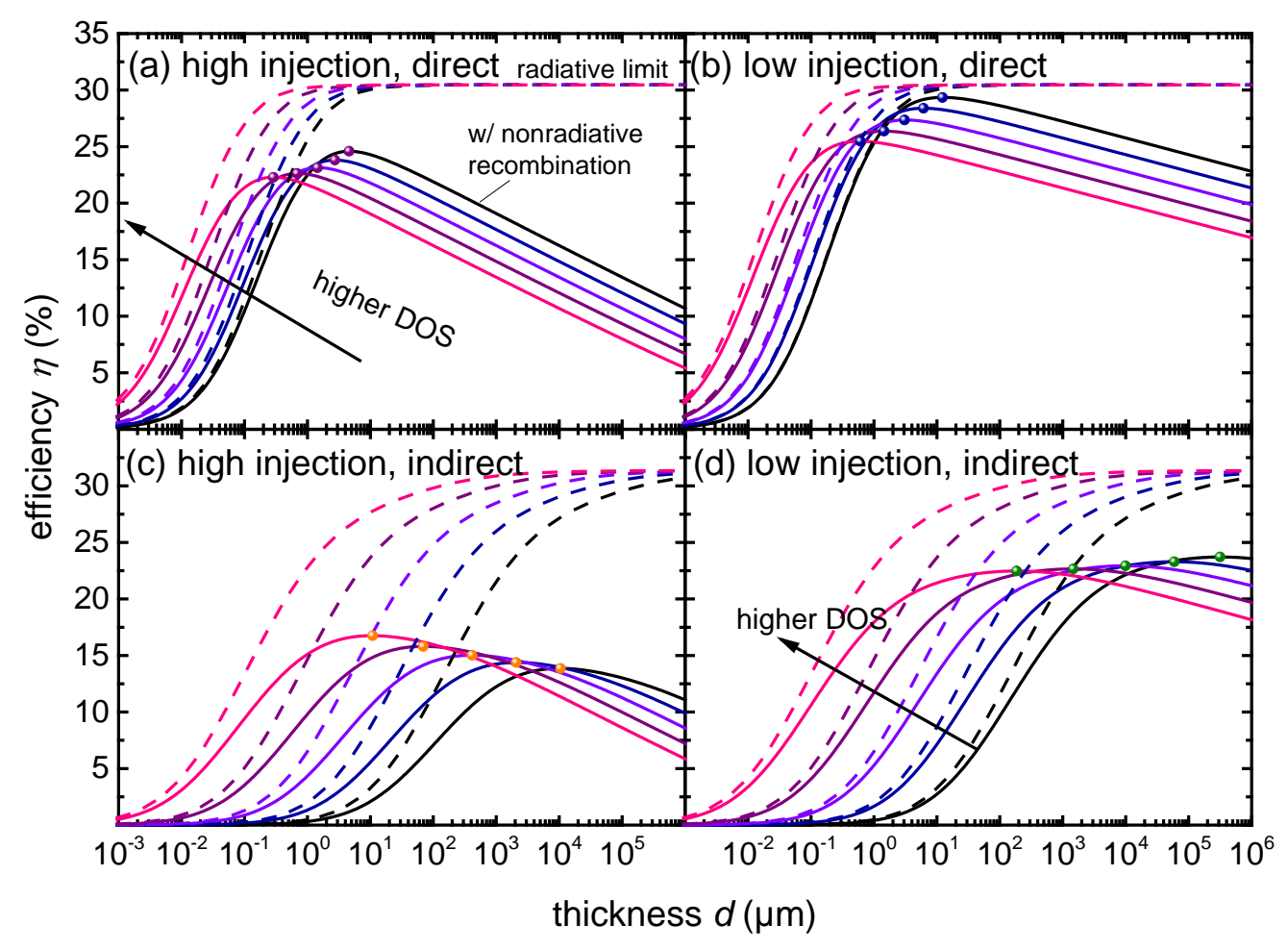

Figure 1: Simulated photovoltaic efficiency as a function of thickness and with the effective density of states as parameter $\left(N_{\text {eff }}=10^{18}, 10^{18.5}, 10^{19}, 10^{19.5}, 10^{20} \mathrm{~cm}^{-3}\right)$ for four different cases: The cases are all combinations of high and low level injection (intrinsic and doped semiconductor) and direct and indirect band gap semiconductors. The parameters for the absorption coefficient are described in table SII and the SRH lifetime is $\tau_{\mathrm{n}}=\tau_{\mathrm{p}}=1 \mu \mathrm{s}$. The dashed lines represent the radiative limit and saturates at high thicknesses towards the SQlimit at a band gap of $1.6 \mathrm{eV}\left(\eta_{\mathrm{SQ}}=30.5 \%\right)$. The solid lines also consider non-radiative recombination leading to lower $V_{\mathrm{oc}}$ at high thicknesses (c.f. Fig. S2) and therefore the efficiency reaches a maximum at a finite optimum thickness (closed symbols, also see Fig. 2). Note the corresponding range of effective masses is $0.11 \mathrm{~m}$ up to $2.53 \mathrm{~m}$, where $\mathrm{m}$ is the free electron mass. 


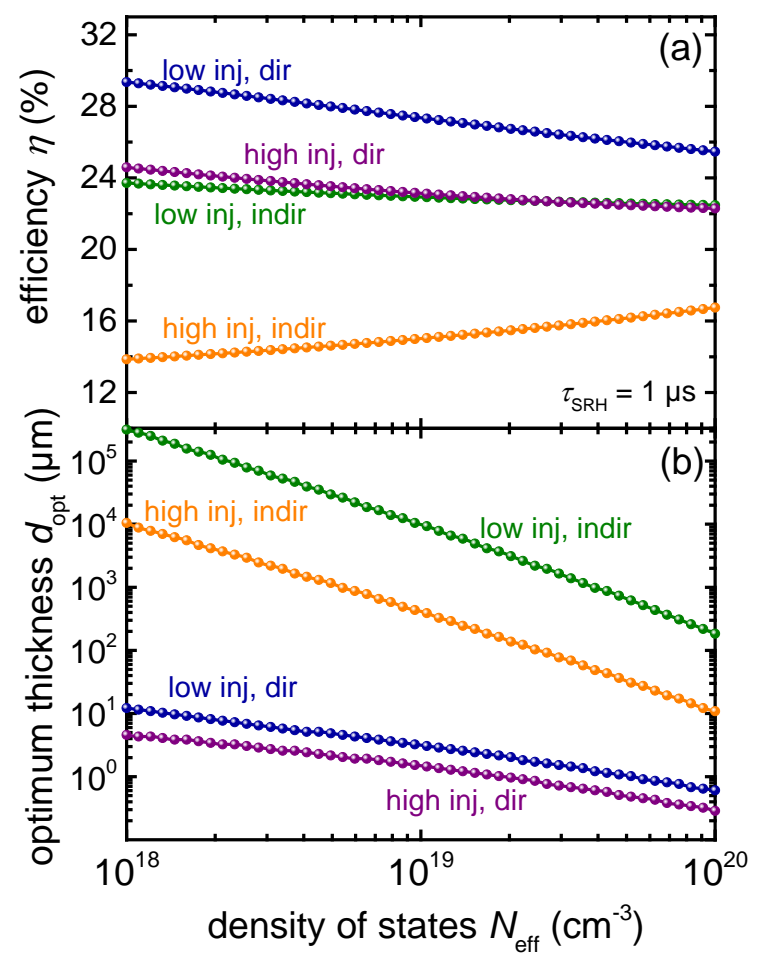

Figure 2: (a) Optimum efficiency and (b) the corresponding optimum thickness as a function of effective density of states for the four cases discussed in Fig. 1. The data includes nonradiative recombination and is consistent with the symbols in Fig. 1 but more data points are used for the calculation. 


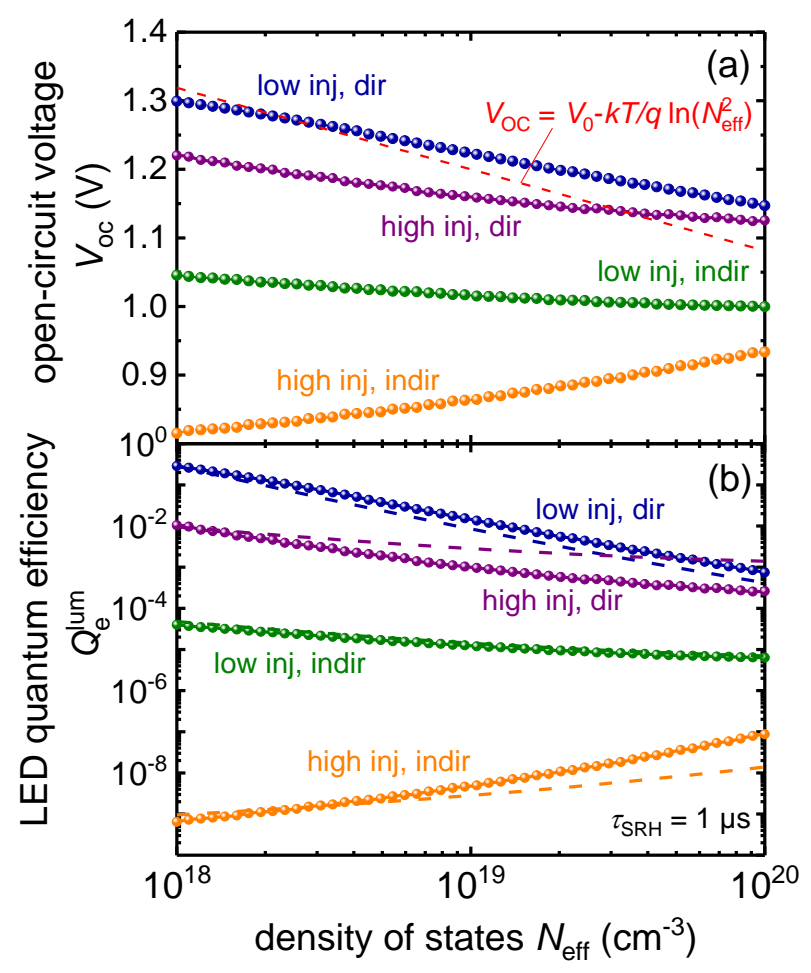

Figure 3: (a) Open-circuit voltage and (b) external LED quantum efficiency at the point of optimum photovoltaic efficiency as a function of effective density of states for the same four cases as discussed in Figs. 1 and 2. The symbols indicate the data from the simulations and the dashed lines in (a) represent the simplistic approximation in Eq. (3) (with $n p=$ const) that does not reproduce the data while the dashed lines in panel (b) represent the analytical predictions for the internal LED quantum efficiency given in table II that do reproduce the different trends rather well. By comparison between Fig. 3 and Fig. 2a it becomes clear that the trend of efficiency vs. effective DOS is mostly controlled by the trend in $V_{\text {oc }}$ at the optimum thickness while $J_{\text {sc }}$ varies from case to case but remains mostly constant with $N_{\text {eff }}$ within one scenario (see Fig. S3). 


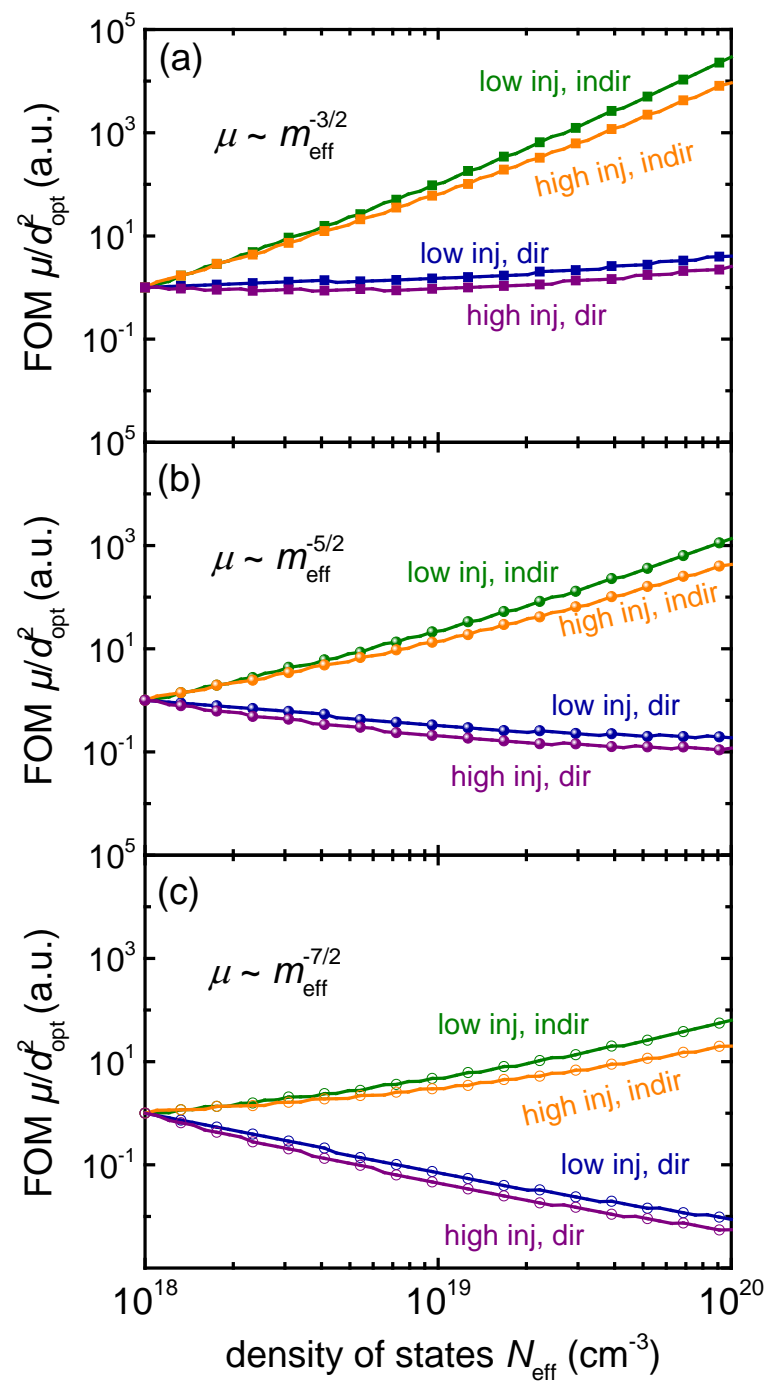

Figure 4: Dependence of the figure of merit $\mu / d_{\mathrm{opt}}^{2}$ for charge collection on the effective density of states for three different functional dependencies of the mobility on the effective mass: (a) $\mu \propto m_{\text {eff }}^{-3 / 2}$, (b) $\mu \propto m_{\text {eff }}^{-5 / 2}$, and (c) $\mu \propto m_{\text {eff }}^{-7 / 2}$. While lower $m_{\text {eff }}$ means higher mobility, it also means higher optimum thickness and therefore, the ratio may increase or decrease with $N_{\text {eff }}$ or $m_{\text {eff }}$ depending on the dominant scattering model and the type of band gap. Low injection vs. high injection is however not a strong factor. 


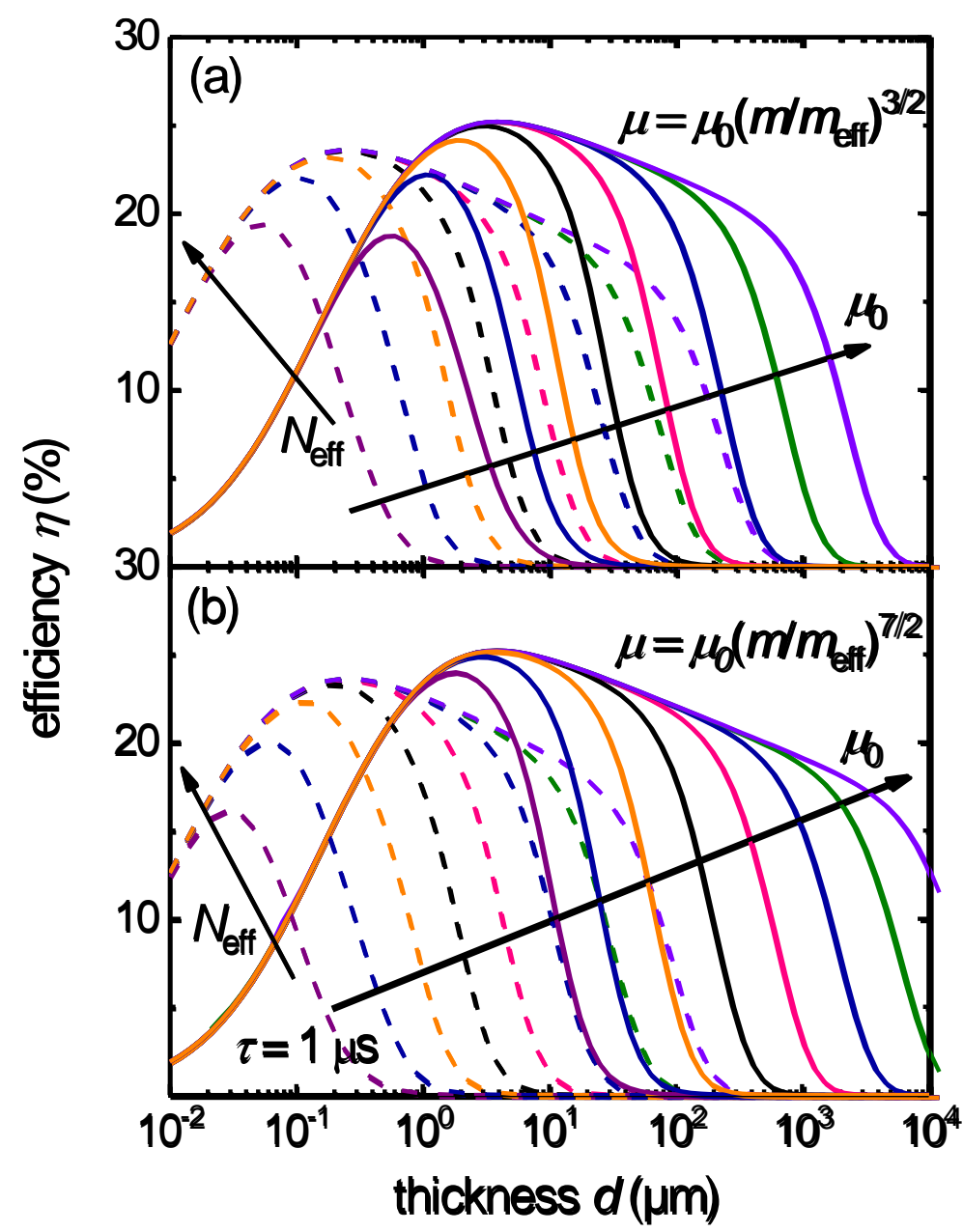

Figure 5: Numerical drift-diffusion simulations as a function of thickness for the case of a direct semiconductor in high level injection for two different effective densities of states $\left(N_{\text {eff }}\right.$ $=10^{18}$ and $10^{20} \mathrm{~cm}^{-3}$ ), two different scattering models ((a) $\mu \propto m_{\text {eff }}^{-3 / 2}$, (b) $\mu \propto m_{\text {eff }}^{-7 / 2}$ ) and 5 different mobility prefactors $\left(\mu_{0}=10^{-3}, 10^{-2}, \ldots 10^{3}, 10^{4} \mathrm{~cm}^{2} / \mathrm{Vs}\right)$. The data for high mobilities corresponds approximately to Fig. 1a (black and pink line) which deals with the infinite mobility case in an analytical approximation. In the case of (a), the FOM in Fig. 4a is nearly identical for both $N_{\text {eff }}$ values. Thus the effect of lowering $\mu_{0}$ is similar but shifted in thickness. In the case of (b) the FOM in Fig. 4c is much higher (better) in case of the low $N_{\text {eff, }}$ thus we notice a broader shoulder for the low $N_{\text {eff }}$ (solid lines) and a shallower one for the high $N_{\text {eff }}$ (dashed lines). 


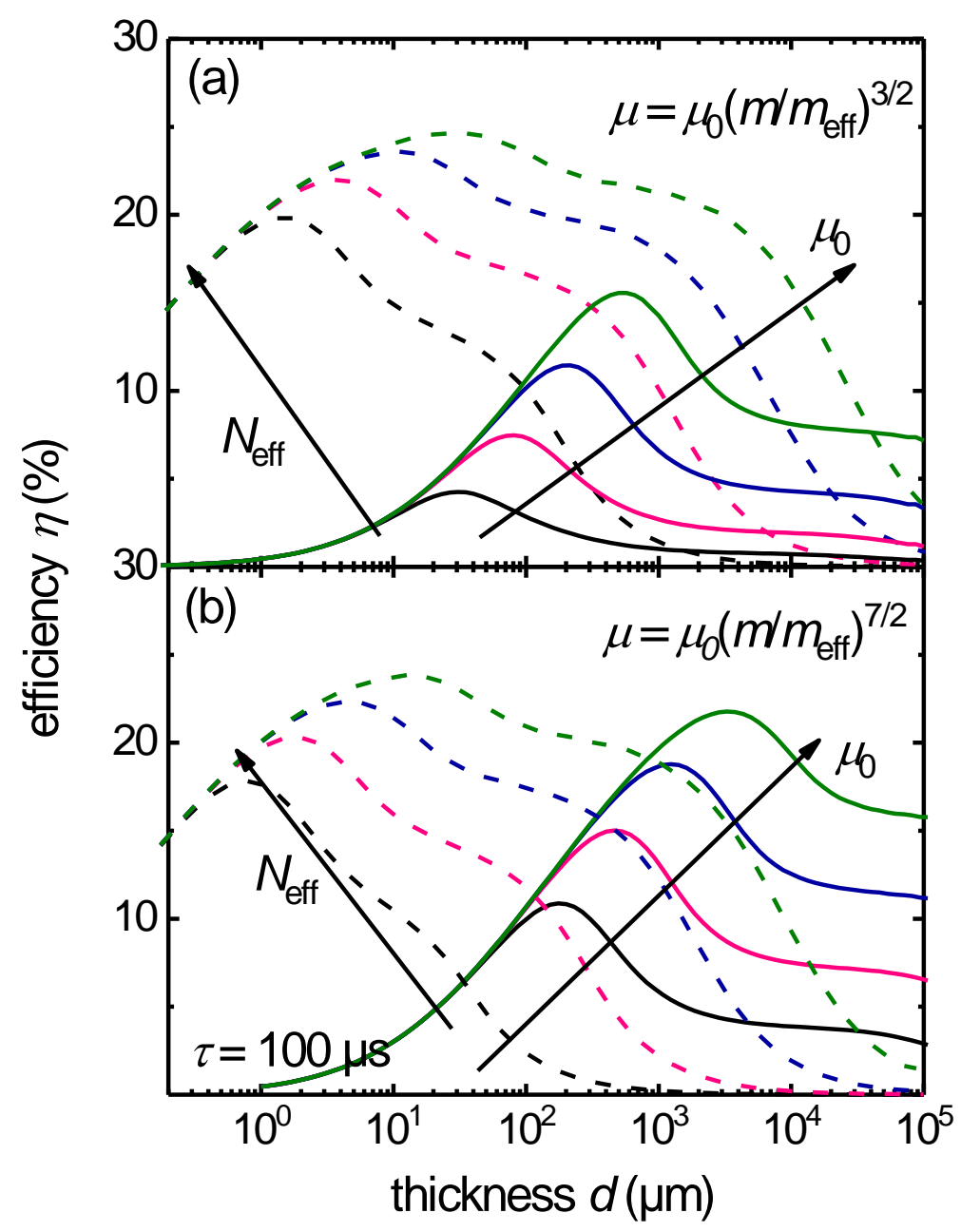

Figure 6: Numerical drift-diffusion simulations as a function of thickness for the case of an indirect doped semiconductor $\left(N_{\mathrm{dop}}=10^{17} \mathrm{~cm}^{-3}\right.$ ) for two different effective densities of states ( $N_{\text {eff }}=10^{18}$ and $10^{20} \mathrm{~cm}^{-3}$ ), two different scattering models ((a) $\mu \propto m_{\text {eff }}^{-3 / 2}$, (b) $\mu \propto m_{\text {eff }}^{-7 / 2}$ ) and 4 different mobility prefactors $\left(\mu_{0}=10^{-1}, 10^{0}, 10^{1}, 10^{2} \mathrm{~cm}^{2} / \mathrm{Vs}\right)$. The lifetime was set to $100 \mu \mathrm{s}$ to keep the mobilities necessary for efficient collection at the relatively higher thicknesses in a realistic range. The corresponding FOMs are given by the green lines in Figs. 4a and 4c. The FOMs explain why in the case displayed in panel (a), the high effective DOS is substantially more beneficial than the lower DOS. In panel (b) the high DOS is still better but only by a small margin. 
Reference List

1. M. A. Green, A. Ho-Baillie, and H. J. Snaith, Nature Photonics, 2014, 8, 506.

2. S. D. Stranks and H. J. Snaith, Nature Nanotechnology, 2015, 10, 391.

3. D. P. McMeekin, G. Sadoughi, W. Rehman, G. E. Eperon, M. Saliba, M. T. Hörantner, A. Haghighirad, N. Sakai, L. Korte, B. Rech, M. B. Johnston, L. M. Herz, and H. J. Snaith, Science, 2016, 351, 151.

4. W. Zhang, G. E. Eperon, and H. J. Snaith, Nature Energy, 2016, 1, 16048.

5. N. J. Jeon, J. H. Noh, W. S. Yang, Y. C. Kim, S. Ryu, J. Seo, and S. I. Seok, Nature, 2015, 517, 476.

6. W. S. Yang, J. H. Noh, N. J. Jeon, Y. C. Kim, S. Ryu, J. Seo, and S. I. Seok, Science, 2015, 348, 1234.

7. F. Deschler, M. Price, S. Pathak, L. E. Klintberg, D. D. Jarausch, R. Higler, S. Huttner, T. Leijtens, S. D. Stranks, H. J. Snaith, M. Atature, R. T. Phillips, and R. H. Friend, Journal of Physical Chemistry Letters, 2014, 5, 1421.

8. Z. K. Tan, R. S. Moghaddam, M. L. Lai, P. Docampo, R. Higler, F. Deschler, M. Price, A. Sadhanala, L. M. Pazos, D. Credgington, F. Hanusch, T. Bein, H. J. Snaith, and R. H. Friend, Nature Nanotechnology, 2014, 9, 687.

9. H. Cho, S. H. Jeong, M. H. Park, Y. H. Kim, C. Wolf, C. L. Lee, J. H. Heo, A. Sadhanala, N. Myoung, S. Yoo, S. H. Im, R. H. Friend, and T. W. Lee, Science, 2015, 350, 1222.

10. B. R. Sutherland and E. H. Sargent, Nature Photonics, 2016, 10, 295.

11. G. Lanzani, A. Petrozza, and M. Caironi, Nature Photonics, 2017, 11, 20.

12. S. Colella, M. Mazzeo, A. Rizzo, G. Gigli, and A. Listorti, Journal of Physical Chemistry Letters, 2016, 7, 4322.

13. M. Saliba, T. Matsui, K. Domanski, J. Y. Seo, A. Ummadisingu, S. M. Zakeeruddin, J. P. Correa-Baena, W. R. Tress, A. Abate, A. Hagfeldt, and M. Grätzel, Science, 2016, 354, 206.

14. M. Saliba, T. Matsui, J. Y. Seo, K. Domanski, J. P. Correa-Baena, M. K. Nazeeruddin, S. M. Zakeeruddin, W. Tress, A. Abate, A. Hagfeldt, and M. Gratzel, Energy \& Environmental Science, 2016, 9, 1989.

15. M. Saliba, S. Orlandi, T. Matsui, S. Aghazada, M. Cavazzini, J. P. Correa-Baena, P. Gao, R. Scopelliti, E. Mosconi, and K. H. Dahmen, Nature Energy, 201615017. 
16. K. T. Cho, S. Paek, G. Grancini, C. Roldan-Carmona, P. Gao, Y. Lee, and M. K. Nazeeruddin, Energy \& Environmental Science, 2017, 10, 621.

17. W. J. Yin, T. T. Shi, and Y. F. Yan, Applied Physics Letters, 2014, 104, 063903.

18. D. A. Egger, A. Bera, D. Cahen, G. Hodes, T. Kirchartz, L. Kronik, R. Lovrincic, A. M. Rappe, D. R. Reichman, and O. Yaffe, Advanced Materials, 2018, 0, 1800691.

19. M. A. Green, K. Emery, Y. Hishikawa, W. Warta, and E. D. Dunlop, Progress in Photovoltaics: Research and Applications, 2016, 24, 3.

20. L. P. Yu, S. Lany, R. Kykyneshi, V. Jieratum, R. Ravichandran, B. Pelatt, E. Altschul, H. A. S. Platt, J. F. Wager, D. A. Keszler, and A. Zunger, Advanced Energy Materials, 2011, 1, 748.

21. L. Yu and A. Zunger, Physical Review Letters, 2012, 108, 068701.

22. R. E. Brandt, V. Stevanoviç, D. S. Ginley, and T. Buonassisi, MRS Communications, 2015, 5, 265.

23. L. M. Ghiringhelli, J. Vybiral, S. V. Levchenko, C. Draxl, and M. Scheffler, Physical Review Letters, 2015, 114, 105503.

24. B. Blank, T. Kirchartz, S. Lany, and U. Rau, Physical Review Applied, 2017, 8, 024032.

25. T. Kirchartz and U. Rau, Advanced Energy Materials, 2018, 0, 1703385.

26. F. Giustino and H. J. Snaith, ACS Energy Letters, 2016, 1, 1233.

27. A. Miyata, A. Mitioglu, P. Plochocka, O. Portugall, J. T.-W. Wang, S. D. Stranks, H. J. Snaith, and R. J. Nicholas, Nat.Phys., 2015, 11, 582.

28. F. Staub, H. Hempel, J. C. Hebig, J. Mock, U. W. Paetzold, U. Rau, T. Unold, and T. Kirchartz, Physical Review Applied, 2016, 6, 044017.

29. G. Giorgi, J. I. Fujisawa, H. Segawa, and K. Yamashita, Journal of Physical Chemistry Letters, 2013, 4, 4213.

30. F. Brivio, K. T. Butler, A. Walsh, and M. van Schilfgaarde, Physical Review B, 2014, 89, 155204.

31. N. Ashari-Astani, S. Meloni, A. H. Salavati, G. Palermo, M. Gr+ñtzel, and U. Rothlisberger, The Journal of Physical Chemistry C, 2017, 121, 23886.

32. Y. Zhou and G. Long, The Journal of Physical Chemistry C, 2017, 121, 1455.

33. E. M. Hutter, M. C. Gelvez-Rueda, A. Osherov, V. Bulovic, F. C. Grozema, S. D. Stranks, and T. J. Savenije, Nature Materials, 2017, 16, 115.

34. J. E. Moser, Nature Materials, 2017, 16, 4. 
35. T. Wang, B. Daiber, J. M. Frost, S. A. Mann, E. C. Garnett, A. Walsh, and B. Ehrler, Energy \& Environmental Science, 2017, 10, 509.

36. T. Kirchartz and U. Rau, The Journal of Physical Chemistry Letters, 2017, 8, 1265.

37. S. M. Sze, in Physics of Semiconductor Devices, John Wiley \& Sons, New York, 2nd Edition ed., 1981, pp. 17.

38. B. K. Ridley, in Quantum Processes in Semiconductors, Oxford University Press, Oxford, 2013, pp. 173.

39. W. van Roosbroeck and W. Shockley, Physical Review, 1954, 94, 1558.

40. W. Shockley and W. T. Read, Physical Review, 1952, 87, 835.

41. R. N. Hall, Physical Review, 1952, 87, 387.

42. S. M. Sze, in Physics of Semiconductor Devices, John Wiley \& Sons, New York, 2nd Edition ed., 1981, pp. 35.

43. T. Markvart, in Recombination in Semiconductors, ed.P. T. Landsberg, Cambridge University Press, Cambridge, 2003, pp. 474.

44. M. A. Green, in Solar cells, operating principles, technology and system applications, University of New South Wales, 1986, pp. 79.

45. Reference Solar Spectral Irradiance: Air Mass 1.5. 2017. NREL, Golden Colorado, American Society for Testing and Materials.

Ref Type: Online Source

46. U. Rau, Physical Review B, 2007, 76, 085303.

47. P. Würfel, Journal of Physics C-Solid State Physics, 1982, 15, 3967.

48. M. A. Green, Progress in Photovoltaics, 2002, 10, 235.

49. O. J. Sandberg, A. Sundqvist, M. Nyman, and R. Österbacka, Physical Review Applied, 2016, 5, 044005.

50. I. Zonno, B. Krogmeier, V. Katte, D. Lübke, A. Martinez-Otero, and T. Kirchartz, Applied Physics Letters, 2016, 109, 183301.

51. W. Shockley and H. J. Queisser, Journal of Applied Physics, 1961, 32, 510.

52. R. Brendel and H. J. Queisser, Solar Energy Materials and Solar Cells, 1993, 29, 397.

53. T. Kirchartz, F. Staub, and U. Rau, ACS Energy Letters, 2016, 1, 731.

54. T. Kirchartz, J. Bisquert, I. Mora-Sero, and G. Garcia-Belmonte, Physical Chemistry Chemical Physics, 2015, 17, 4007.

55. U. Rau, U. W. Paetzold, and T. Kirchartz, Physical Review B, 2014, 90, 035211.

56. U. Rau and T. Kirchartz, Nature Materials, 2014, 13, 103. 
57. B. K. Ridley, in Quantum Processes in Semiconductors, Oxford University Press, Oxford, 2013, pp. 109.

58. N. D. Arora, S. G. Chamberlain, and D. J. Roulston, Applied Physics Letters, 1980, 37, 325.

59. T. Kirchartz, A. Helbig, and U. Rau, Solar Energy Materials and Solar Cells, 2008, 92, 1621.

60. R. S. Crandall, Journal of Applied Physics, 1982, 53, 3350.

61. R. S. Crandall, Journal of Applied Physics, 1983, 54, 7176.

62. R. A. Street, M. Schoendorf, A. Roy, and J. H. Lee, Physical Review B, 2010, 81, 205307.

63. P. Kaienburg, U. Rau, and T. Kirchartz, Physical Review Applied, 2016, 6, 024001.

64. D. Bartesaghi, I. d. C. Perez, J. Kniepert, S. Roland, M. Turbiez, D. Neher, and L. J. A. Koster, Nature Communications, 2015, 6, 7083.

65. B. E. Pieters, J. Krc, and M. Zeman, Advanced numerical simulation tool for solar cells - ASA5, Conference Record of the 2006 IEEE 4th World Conference on Photovoltaic Energy Conversion, 2006.

66. B. E. Pieters, K. Decock, M. Burgelman, R. Stangl, and T. Kirchartz, in Advanced Characterization Techniques for Thin Film Solar Cells, ed.D. Abou-Ras, T. Kirchartz, U. Rau, Wiley-VCH Verlag GmbH \& Co. KGaA, 2011, pp. 501-527.

67. S. B. Zhang, S. H. Wei, A. Zunger, and H. Katayama-Yoshida, Physical Review B, 1998, 57, 9642.

68. W. J. Yin, T. Shi, and Y. Yan, Applied Physics Letters, 2014, 104, 063903.

69. M. Sendner, P. K. Nayak, D. A. Egger, S. Beck, C. Muller, B. Epding, W. Kowalsky, L. Kronik, H. J. Snaith, A. Pucci, and R. Lovrincic, Materials Horizons, 2016, 3, 613.

70. T. Kirchartz, T. Markvart, U. Rau, and D. A. Egger, The Journal of Physical Chemistry Letters, 2018, 9, 939.

71. K. Huang and A. Rhys, Proceedings of the Royal Society of London.Series A.Mathematical and Physical Sciences, 1950, 204, 406.

72. B. K. Ridley, in Quantum Processes in Semiconductors, Oxford University Press, Oxford, 2013, pp. 224.

73. B. K. Ridley, in Quantum Processes in Semiconductors, Oxford University Press, Oxford, 2013, pp. 168.

74. B. K. Ridley, in Quantum Processes in Semiconductors, Oxford University Press, Oxford, 2013, pp. 186.

75. T. Markvart, Journal of Physics C: Solid State Physics, 1981, 14, L895. 
76. T. Markvart, in Recombination in Semiconductors, ed.P. T. Landsberg, Cambridge University Press, Cambridge, 2003, pp. 467-468. 